\title{
Model-Based Fault Diagnosis Approach for Rotor System with Unidentified Supporting Parameters
}

\author{
Hongliang Yao ${ }^{1, a}$, Hongbin Ma,b ${ }^{2, b}$ Qingkai Han ${ }^{1, c}$ and Bangchun Wen ${ }^{1, d}$ \\ ${ }^{1}$ School of Mechanical Engineering and Automation, Northeastern University, Shenyang, PR China, \\ ${ }^{2}$ Bethune International Peace Hospital of PLA, Shijiazhuang, PR China \\ ahlyao@mail.neu.edu.cn(corresponding author), bmahongbin1977@gmail.com, \\ cqhan@mail.neu.edu.cn, dbcwen1930@vip.sina.com
}

\begin{abstract}
Keywords: Rotating machinery, Model based diagnosis method, Fixed-interface modal synthesis method
\end{abstract}

\begin{abstract}
The model based diagnosis method can detect both the fault location and the fault extent of the rotating machinery at the same time as it can combine the dynamics and fault diagnosis method together. However, the modal test of the supporting part is necessary when using the existing model based method, and the precision of the modal test results make a strong impact on the diagnosis results. In order to improve this case, an improved model based diagnosis method is presented herein by combining the fixed-interface modal synthesis method with the traditional model based diagnosis method. The DOF of the unidentified parameter due to the supporting parts are partitioned to interface DOF, and the displacement information of the supporting parts can be measured by the mounted sensors. Therefore, the model based diagnosis method can be carried out by only using the parameters of the rotor without considering the effects from the supporting, which can greatly improve the diagnosis precision and efficiency. This method is fit for the rotor systems with unidentified supporting parameter, and the rotor systems with unknown nonlinear supporting parameter. Both the numerical simulation results and the experiment results in the example proved the efficiency of this method.
\end{abstract}

\section{Introduction}

The study of fault diagnosis is always an active topic in rotor dynamics, due to the fact that it is capable of providing early indication of the impending failure of the operating large scale rotating machinery ${ }^{[1]}$. Online detection and detailed information about the fault location and fault severity becomes more vital as the actual rotating machinery operates on higher speed and may cause catastrophic damage when faults happen. The conventional fault diagnosis methods based on signals are efficient on identifying fault types, yet it gives only qualitative and limited information about the location and extent of fault. The model based identification method, on the contrary, can identify the fault location and fault extent as it utilized the dynamic information of the rotor system during the process of identification.

The model based diagnosis methods appear in the late 90 s of 20th century. There are three types of model based fault diagnosis method for rotating machinery at present. The first type is in time domain and presented by Markert et al. ${ }^{[2]}$. The modal expansion technique is applied to estimate the full vibrational state of the rotor system and the equivalent loads of the fault is obtained by using the least-squares fitting algorithm. The equivalent loads give information about the fault locations and fault extent. Sekhar ${ }^{[3,4]}$ used this method to identify the single and multi-cracks in the shaft, Jain and Kundra $^{[5]}$ applied this method to detect unbalance and crack, Jalan and Mohanty ${ }^{[6]}$ applied this method to detect misalignment and unbalance, Yao et al. ${ }^{[7]}$ used this method to study the local rub faults of rotor system. This method is very easy to apply, but the estimated vibrations of the whole rotor system may differ much to the actual vibrations as the mode truncation in modal expansion technique can cause error.

Another more accurate model based method in frequency domain is presented and developed by Bachschmid, Pennacchi and Vania ${ }^{[8-10]}$. In this method, the finite element model of the rotor system is built and the possible fault locations are hypothesized, the harmonic components of equivalent force or moment are obtained by comparing the vibrations of the fault system with the reference system 
(usually normal operating rotor system), then the fault location and fault extent can be identified by least-squares fitting approach. This method is proved to be efficient in detecting single fault or multi-fault as it has been applied on the identification of all kinds of faults, both in test-rigs and in actual large scale rotating machines. For example, in reference ${ }^{[8]}$ the detecting methods and fault mathematic models are introduced and common faults such as unbalance, crack and rotor to stator rub are identified in test-rig; Pennacchi et al..$^{[9,10]}$ applied this method to detect crack fault of a test rig and the thermal bow of a 50MW power unit; Han et al. ${ }^{[1]}$ used a method alike to identify the unbalance of the rotor system. This method gives more accuracy since the modal expansion is avoided.

The other model based identification method is the system identification method and is also carried out in the frequency domain. Edwards and Lees applied this method to detect unbalance and misalignment faults and so on ${ }^{[12-14]}$. The characteristic of this method is that the mathematical model of the system is the full rotor-bearing-supporting model, and the dynamic stiffness matrix is used to identify the faults.

The aforementioned methods have been applied to every kind of common faults of rotating machinery, which have proved the efficiency of the model based identification method. However, all the methods mentioned above need an accurate model of the rotor system, including the rotor dimensions, the supporting stiffness and so on. To know all the parameters of the rotor system, the modal testing must be carried out, which is difficult and with low precision. The supporting parts of the field large scale rotating machinery are usually sliding bearings, the stiffness and damping coefficients of which change nonlinearly with the rotating speed. Thus, the identification of the stiffness and damping coefficients is very difficult and usually need complicated procedures, which may result in unsatisfied results. These defects greatly restrict the development and popularization of the model based identification method.

In this paper, a novel model based identification method is provided. The fixed-interface modal synthesis method, which is often used in the structure dynamic analysis to separate nonlinear degrees from linear degrees, is introduced into the model based identification method to divide the DOF of unidentified parameter supporting parts to interface DOF. The other parts of the rotor system, which with known parameters are divided to interior DOF. Then the finite element model of the rotor system is built up by only the parts with known parameter. In the identification process, the displacement information of the supporting parts can be measured from mounted sensors. The least-squares fitting approach is used to identify the fault location and fault severity. By this method, the model based diagnosis method is carried out by only using the parameters of the rotor, not the supporting, which can greatly improve the precision and the efficiency and mimic the condition of actual rotating machinery. The numerical simulation results and the experiment results proved the efficiency of this method.

\section{The principle and procedure of the model based identification method}

Principle and procedure of the traditional model based method ${ }^{[8-10]}$. The model based identification is to combine the dynamics of the rotor system with the common signal based identification method. The vibration difference between the fault rotor system and the normal rotor system is combined with the mathematical model of the rotor system.

The standard matrix equation of the normally operating rotor system is

$\boldsymbol{M} \ddot{\boldsymbol{X}}+\boldsymbol{C} \dot{\boldsymbol{X}}+\boldsymbol{K} \boldsymbol{X}=\boldsymbol{F}$

The occurrence of a fault will change the rotor system's dynamic behavior. The fault-induced change in the vibrational behavior is represented by the residual vibrations on the left side and additional force on the right hand

$\boldsymbol{M}(\ddot{\boldsymbol{X}}+\mathrm{d} \ddot{\boldsymbol{X}})+\boldsymbol{C}(\dot{\boldsymbol{X}}+\mathrm{d} \dot{\boldsymbol{X}})+\boldsymbol{K}(\boldsymbol{X}+\mathrm{d} \boldsymbol{X})=\boldsymbol{F}+\Delta \boldsymbol{F}$

Substitute (1) into (2) will yield

$\boldsymbol{M} \cdot \mathrm{d} \ddot{\boldsymbol{X}}+\boldsymbol{C} \cdot \mathrm{d} \dot{\boldsymbol{X}}+\boldsymbol{K} \cdot \mathrm{d} \boldsymbol{X}=\Delta \boldsymbol{F}$

Thus, the fault resulted force (equivalent loads) can be considered as a separate force vector working on the un-fault rotor system. 
For the actual rotating machinery, only a few sensors are located to detect the vibration of the rotor system. Assuming there are $m$ measuring nodes existing in the $n$-Node rotor system, the measuring locations are $\mathrm{i}, \mathrm{j}, \mathrm{k}$, and the measuring matrix is: $\boldsymbol{X}_{\mathrm{m}}=\boldsymbol{T} \boldsymbol{X}$

The form of matrix $\mathrm{T}$ is described as follows:

$\left[\begin{array}{ccc}\underbrace{0 \cdots 0}_{i-1} & 1 & 0 \cdots 0 \\ \underbrace{0 \cdots 0}_{j-1} & 1 & 0 \cdots 0 \\ \vdots & \vdots & \vdots \\ \underbrace{0 \cdots 0}_{k-1} & 1 & 0 \cdots 0\end{array}\right]_{\mathrm{m} \times \mathrm{N}}$

Assuming the difference of the vibrations of fault and un-fault rotor system is $\boldsymbol{X}_{\mathrm{m}}=\boldsymbol{X}_{\mathrm{m} 2}-\boldsymbol{X}_{\mathrm{m} 1}$. Here, $\boldsymbol{X}_{\mathrm{m} 1}$ is the vibrations of the un-fault rotor system, $\boldsymbol{X}_{\mathrm{m} 2}$ is the vibration of the fault rotor system. Assuming the fault force vector $\boldsymbol{F}(\Omega)$, and applying the harmonic balance criteria, one gets, for the nth harmonic component, the following equation

$\left[-(n \Omega)^{2} \boldsymbol{M}+j n \Omega \boldsymbol{C}+\boldsymbol{K}\right] \boldsymbol{X}=\boldsymbol{E}(n \Omega) \boldsymbol{X}=\boldsymbol{F}(\Omega)$

The above equation can be transformed to:

$\left[-(n \Omega)^{2} \boldsymbol{M}+j n \Omega \boldsymbol{C}+\boldsymbol{K}\right]^{-1} \boldsymbol{F}(\Omega)$

$=\boldsymbol{E}(n \Omega)^{-1} \boldsymbol{F}(\Omega)=\boldsymbol{X}$

A scalar relative residual may be defined by the following equation

$\delta=\left(\frac{\left[\boldsymbol{I} \boldsymbol{E}(n \Omega)^{-1} \boldsymbol{F}(\Omega)-\boldsymbol{X}_{\mathrm{m}}\right]^{\mathrm{T}}\left[\boldsymbol{T} \boldsymbol{E}(n \Omega)^{-1} \boldsymbol{F}(\Omega)-\boldsymbol{X}_{\mathrm{m}}\right]}{\boldsymbol{X}_{\mathrm{m}}^{\mathrm{T}} \boldsymbol{X}_{\mathrm{m}}}\right)^{\frac{1}{2}}$

On the assumption that the fault will happen on node $1,2, \ldots, \mathrm{N}$ and the equivalent force to be $\boldsymbol{F}(\Omega)$, the relative residual $\delta_{i}$ of the least square method can be obtained, the location of the node with the minimum $\delta_{i}$ is the location that fault happens. Then, the detail information of the $\boldsymbol{F}(\Omega)$ can be obtained.

Improved model based method. The above method works very well with the rotor system whose accurate FE model has been obtained. However, for the rotor system whose supporting parameters hasn't been identified, this method is not available. It's difficult to obtain the accurate supporting stiffness of the sliding bearings of large scale rotating machinery and the supporting stiffness may change with rotating speed, temperature and some other environment factors. Hence, the model based method must be improved to handle this problem.

To overcome this shortcoming, the fixed-interface modal synthesis method is combined with the traditional model based method.

By using the fixed-interface modal synthesis method, the DOF of the unidentified parameter supporting parts are partitioned to interface DOF, the dynamic equation of the rotor system changes to:

$$
\begin{aligned}
& {\left[\begin{array}{ll}
\boldsymbol{M}_{\mathrm{ii}} & \boldsymbol{M}_{\mathrm{ij}} \\
\boldsymbol{M}_{\mathrm{ji}} & \boldsymbol{M}_{\mathrm{jj}}
\end{array}\right]\left\{\begin{array}{l}
\ddot{\boldsymbol{X}}_{\mathrm{i}} \\
\ddot{\boldsymbol{X}}_{\mathrm{j}}
\end{array}\right\}+\left[\begin{array}{ll}
\boldsymbol{C}_{\mathrm{ii}} & \boldsymbol{C}_{\mathrm{ij}} \\
\boldsymbol{C}_{\mathrm{ji}} & \boldsymbol{C}_{\mathrm{jj}}
\end{array}\right]\left\{\begin{array}{c}
\dot{\boldsymbol{X}}_{\mathrm{i}} \\
\dot{\boldsymbol{X}}_{\mathrm{j}}
\end{array}\right\}} \\
& +\left[\begin{array}{ll}
\boldsymbol{K}_{\mathrm{ii}} & \boldsymbol{K}_{\mathrm{ij}} \\
\boldsymbol{K}_{\mathrm{ji}} & \boldsymbol{K}_{\mathrm{jj}}
\end{array}\right]\left\{\begin{array}{l}
\boldsymbol{X}_{\mathrm{i}} \\
\boldsymbol{X}_{\mathrm{j}}
\end{array}\right\}=\left\{\begin{array}{l}
\boldsymbol{f}_{\mathrm{i}} \\
\boldsymbol{f}_{\mathrm{j}}
\end{array}\right\}
\end{aligned}
$$

For the steady operating rotor system, each harmonic vibration satisfies harmonic balance equation, which results in:

$$
\left\{\begin{array}{l}
\boldsymbol{E}_{\mathrm{ii}}(n \Omega)=-(n \Omega)^{2} \boldsymbol{M}_{\mathrm{ii}}+j n \Omega \boldsymbol{C}_{\mathrm{ii}}+\boldsymbol{K}_{\mathrm{ii}} \\
\boldsymbol{E}_{\mathrm{ij}}(n \Omega)=-(n \Omega)^{2} \boldsymbol{M}_{\mathrm{ij}}+j n \Omega \boldsymbol{C}_{\mathrm{ij}}+\boldsymbol{K}_{\mathrm{ij}}
\end{array}\right.
$$

It can be obtained from the first row of the former that 


$$
\boldsymbol{E}_{\mathrm{ii}}(n \Omega) \boldsymbol{X}_{\mathrm{i}}+\boldsymbol{E}_{\mathrm{ij}}(n \Omega) \boldsymbol{X}_{\mathrm{j}}=\boldsymbol{F}(\Omega)
$$

which can be changed to:

$$
\boldsymbol{X}_{\mathrm{i}}=\left[\boldsymbol{E}_{\mathrm{ii}}(n \Omega)\right]^{-1} \boldsymbol{F}(\Omega)-\left[\boldsymbol{E}_{\mathrm{ii}}(n \Omega)\right]^{-1} \boldsymbol{E}_{\mathrm{ij}}(n \Omega) \boldsymbol{X}_{\mathrm{j}}
$$

So the least squares fitting method can change to:

$$
\delta=\left(\frac{\left[\boldsymbol{T}\left[\boldsymbol{E}_{\mathrm{ii}}(n \Omega)\right]^{-1} \boldsymbol{F}(\Omega)-\boldsymbol{X}_{\mathrm{m}}^{\prime}\right]^{\mathrm{T}}\left[\boldsymbol{T}\left[\boldsymbol{E}_{\mathrm{ii}}(n \Omega)\right]^{-1} \boldsymbol{F}(\Omega)-\boldsymbol{X}_{\mathrm{m}}^{\prime}\right]}{\left[\boldsymbol{X}_{\mathrm{m}}^{\prime}\right]^{\mathrm{T}} \boldsymbol{X}_{\mathrm{m}}^{\prime}}\right)^{\frac{1}{2}}
$$

where, $\boldsymbol{X}_{\mathrm{m}}^{\prime}=\boldsymbol{T}\left[\boldsymbol{E}_{\mathrm{ii}}(n \Omega)\right]^{-1} \boldsymbol{E}_{\mathrm{ij}}(n \Omega) \boldsymbol{X}_{\mathrm{j}}+\boldsymbol{X}_{\mathrm{m}}$

By this method, the model based diagnosis method can be carried out by only using the parameters of the rotor, not the supporting. In field large scale rotating machinery, there are displacement sensors on supporting places, so it's convenient to obtain the steady response of the supporting. Therefore this method is fit for the rotor systems with unidentified supporting parameter, and it can greatly improve the precision and the efficiency.

Procedure of the model based method. The procedure of the model based method is as follows:

(1) Build the finite element model of the rotor system, the rotor can be meshed into Timoshenko beam elements.

(2) Select a few measuring locations in the rotor. Usually there are displacement sensors on the supporting and some other (often 2-3) sensors are placed on the shaft.

(3) Measure the steady vibration signals of the un-fault rotor system, obtain the amplitude and phase information of the rotor system while using the key-phase signal as a reference.

(4) Obtain the amplitude and phase information of the fault rotor system in the same way as step 3. And then obtain the difference of the amplitude and phase of the fault and un-fault rotor system

(5) Assuming the fault happens on each node, and using equation 7 to obtain the residuals.

(6) The location of the fault can be obtained by comparison of the residuals, and the fault extent can be obtained by the results of the least square method.

\section{Experimental results}

The rotor system used to test the present model based method can be shown as figure 1 . These sensors installing on nodes 4, 12, 19, 1, 21 are used to detect displacements, and the collector is B\&K3560B.

The rotor system is divided into 20 segments with 21 nodes, the lengths, the diameters of each segment is shown in table 1.

Table 1 Lengths and the diameters of the segments

\begin{tabular}{|l|l|l|l|l|l|l|l|l|l|l|l|l|l|l|l|l|l|l|l|l|}
\hline segment & 1 & 2 & 3 & 4 & 5 & 6 & 7 & 8 & 9 & 10 & 11 & 12 & 13 & 14 & 15 & 16 & 17 & 18 & 19 & 20 \\
\hline length $(\mathrm{mm})$ & 20 & 20 & 20 & 20 & 20 & 20 & 20 & 10 & 10 & 20 & 20 & 20 & 20 & 20 & 20 & 20 & 20 & 20 & 20 & 20 \\
\hline diameter $(\mathrm{mm})$ & 10 & 10 & 10 & 10 & 10 & 10 & 10 & 80 & 80 & 10 & 10 & 10 & 10 & 10 & 10 & 10 & 10 & 10 & 10 & 10 \\
\hline
\end{tabular}

The sliding bearing of the rotor system is supported by rubber ring. The nonlinearity in the rotor system is due to the rubber material property and the installation precision. The steady response of the rotor system in rotating speed $\omega_{1}=20 \mathrm{~Hz}, \omega_{2}=25 \mathrm{~Hz}, \omega_{3}=30 \mathrm{~Hz}$ are obtained. For example, when rotating speed is $\omega_{1}=20 \mathrm{~Hz}$, the time wave and the frequency spectrum of node 1 are shown in figure 2. From the frequency spectrum analysis, the $2 \mathrm{X}, 3 \mathrm{X}$ harmonic component, which shows the complexity of the supporting stiffness can be seen.

By using apfft, the amplitude and phase information of node 4, 12, 19, 1, 21 of each rotating speed are obtained, for example, the amplitudes and phases of $20 \mathrm{~Hz}$ are shown in table 2. 


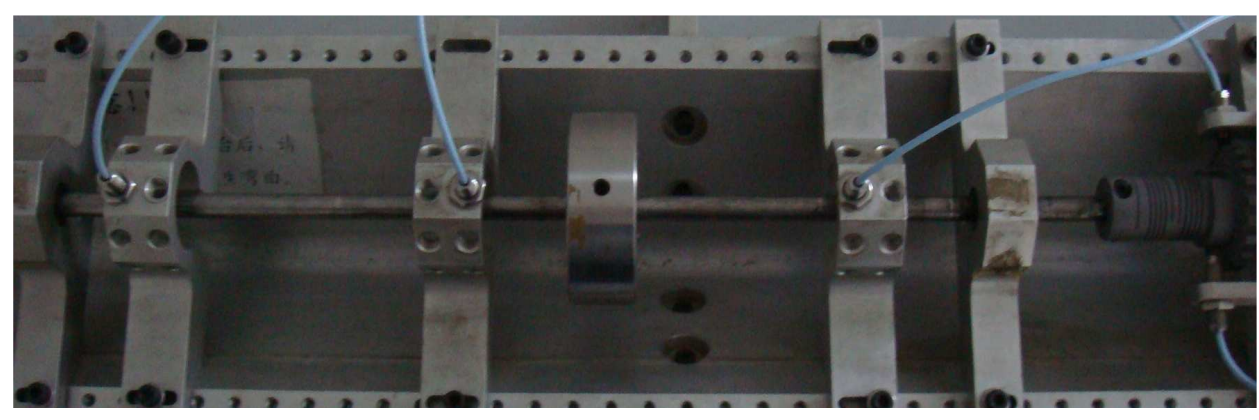

Fig. 1. General view of experimental rotor rig

Table 2 Amplitudes and phases of the measured nodes

\begin{tabular}{|c|c|c|c|c|c|c|c|c|c|c|}
\hline \multirow{2}{*}{} & \multicolumn{2}{|c|}{ node 4 } & \multicolumn{2}{c|}{ node 12 } & \multicolumn{2}{c|}{ node 18 } & \multicolumn{2}{c|}{ node 1 } & \multicolumn{2}{c|}{ node 20 } \\
\cline { 2 - 10 } & $\begin{array}{c}\text { Amp. } \\
(\mathrm{mm})\end{array}$ & $\begin{array}{c}\text { Phase } \\
(\text { degree })\end{array}$ & $\begin{array}{c}\text { Amp. } \\
(\mathrm{mm})\end{array}$ & $\begin{array}{c}\text { Phase } \\
(\text { degree})\end{array}$ & $\begin{array}{c}\text { Amp. } \\
(\mathrm{mm})\end{array}$ & $\begin{array}{c}\text { Phase } \\
(\text { degree })\end{array}$ & $\begin{array}{c}\text { Amp. } \\
(\mathrm{mm})\end{array}$ & $\begin{array}{c}\text { Phase } \\
(\text { degree})\end{array}$ & $\begin{array}{c}\text { Amp. } \\
(\mathrm{mm})\end{array}$ & $\begin{array}{c}\text { Phase } \\
(\mathrm{degree})\end{array}$ \\
\hline $20 \mathrm{~Hz}$ & 0.0953 & 21.521 & 0.1685 & 10.183 & 0.0653 & 0.147 & 0.0094 & 20.093 & 0.0137 & -6.4534 \\
\hline
\end{tabular}

Put the amplitudes and phase magnitudes into the identification program, using equation (7) to identify the eccentric place and its magnitude. The residuals of each node can be shown in figure 3 . It can be seen that the residual on node 8 is the smallest, which is about 0.302 . The magnitude is $m r=0.00108 \mathrm{~kg} . \mathrm{mm}$. The phase is $\varphi=12.8^{\circ}$. The actual eccentricity should be on node 9 , which is very near the identified node 8 . The experiment showed the efficiency of the identification method.
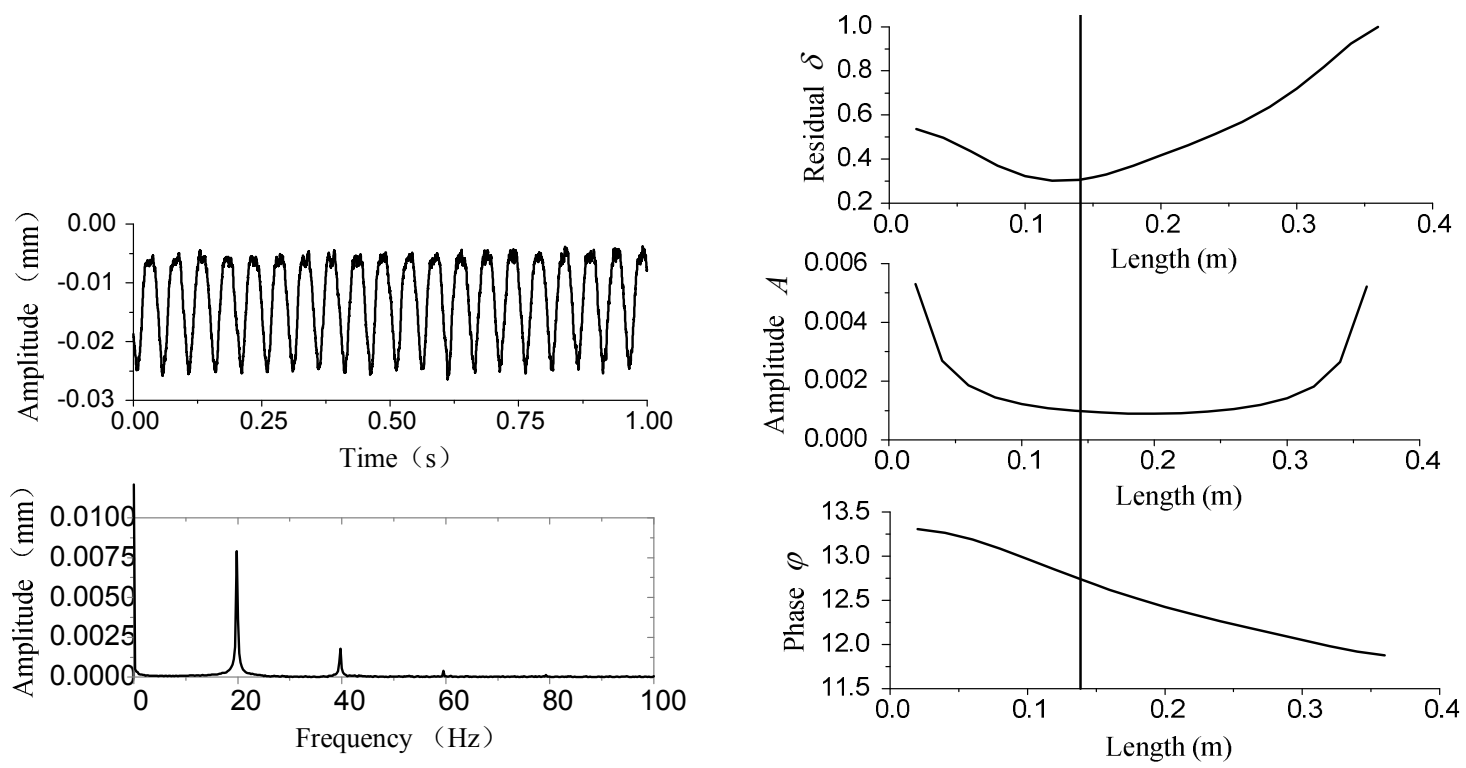

Fig. 2. Measure vibration signal and frequency spectrum when $\omega_{1}=20 \mathrm{~Hz}$

Fig. 3. The experimental results

\section{Conclusions}

A novel model based diagnosis method is presented by combining the fixed-interface modal synthesis method with the traditional model based diagnosis method. The DOF of the unidentified parameter supporting parts are ranked to interface DOF, and the displacement information of the supporting parts can be measured out as it's convenient to mount sensors. By this method, the model based diagnosis method can be carried out by only using the parameters of the rotor, not the supporting, which can greatly improve the precision and the efficiency. 
As the all-phase FFT technique was involved into this method, the identification method becomes more robust to noise disturbing.

The results of the numerical simulation have shown that this method is efficiency. The method has also been applied to experimental results obtained from a test-rig. The test proved the effective in identifying the fault position and extent.

\section{Acknowledgement}

The authors gratefully acknowledge that this work was supported by the Natural Science Foundation of China (grant No. 51005042, 50775028).

\section{References}

[1] Wen, B., et al., The nonlinear dynamic theory and experiment of the fault rotating machinery. Beijing, Science Press (2004) (In chinese)

[2] Markert, R., Platz, R., Seidler, M., Model based fault identification in rotor systems by least square fitting. Proceedings of the 8th international symposium on transport phenomena and dynamics of rotating machinery, ISROMAC-8, Vol. II, 900-906 (2000)

[3] Sekhar, A. S., Crack identification in a rotor system, a model-based approach. Journal of Sound and Vibration. 270 (2004) 887-902

[4] Sekhar, A. S., Model-based identification of two cracks in a rotor system. Mechanical Systems and Signal Processing. 18 (2004) 977-983

[5] Jain, J. R., Kundra, T. K., Model based online diagnosis of unbalance and transverse fatigue crack in rotor systems. Mechanics Research Communications. 31 (2004) 557-568

[6] Jalan, A. K., Mohanty, A. R., Model based fault diagnosis of a rotor-bearing system for misalignment and unbalance under steady-state condition. Journal of Sound and Vibration. 327 (2009) 604-622

[7] Yao, H. L., Li, H., Li, X. P., Wen, B. C., Diagnosis of local fault and identification of transient fault force in rotating machinery. Journal of Mechanical Engineering, 43 (2007) 120-124 (In chinese)

[8] Bachschmid, N., Pennacchi, P., Vania, A., Identification of multiple faults in rotor systems. Journal of Sound and Vibration, 254 (2002) 327-366

[9] Pennacchi, P., Bachschmid, N., Vania, A., A model-based identification method of transverse cracks in rotating shafts suitable for industrial machines. Mechanical Systems and Signal Processing 20 (2006) 2112-2147

[10] Pennacchi, P., Vania, A., Accuracy in the identification of a generator thermal bow. Journal of Sound and Vibration 274 (2004) 273-295

[11] Han, Q. K., Yao, H. L., Wen, B. C., Parameter identification for a rotor system based on its finite element model and with varying speeds. Acta Mech Sin 26 (2010) 299-303

[12] Sinha, J. K., Lees, A .W., Friswell, M. I., Estimating unbalance and misalignment of a flexible rotating machine from a single run-down. Journal of Sound and Vibration. 272 (2004) 967-989

[13] Edwards, S., Lees, A .W., Friswell, M. I., Experimental identification of excitation and support parameters of a flexible rotor-bearings-foundation system from a single run-down. Journal of Sound and Vibration. 232(2000), 63-992

[14] Lees, A. W., Sinha, J. K., Friswell, M. I., Model-based identification of rotating machines. Mechanical Systems and Signal Processing. 23 (2009) 1884-1893 